\title{
Implications of inhomogeneous distribution of concentration polarization for interpretation of pressure-driven membrane measurements
}

\author{
Marc Fernández de Labastida ${ }^{1}$, Edxon E.Licón ${ }^{1}$, Andriy Yaroshchuk ${ }^{1,2}$ \\ ${ }^{1}$ Dept of Chemical Engineering, Polytechnic University of Catalonia - BarcelonaTech, av. \\ Diagonal 647, 08028 Barcelona, Spain \\ ${ }^{2}$ ICREA, Passeig Lluís Companys 23, Barcelona, Spain
}

\begin{abstract}
A number of CFD studies have demonstrated that there is a considerable inhomogeneity of extent of Concentration Polarization (CP) over the membrane surface especially in spacer-filled feed channels. However, the consequences of this inhomogeneity for the interpretation of measurements of solute rejection in pressure-driven membrane processes have received little attention.
\end{abstract}

This study uses a simple model of locally-1D CP combined with a postulated probability distribution of unstirred-layer thickness over the membrane thickness. In this way, we obtain transparent analytical results and can consider qualitative consequences of inhomogeneous distribution of CP over membrane surface. Our analysis shows that disregarding the CP distribution under-estimates the $\mathrm{CP}$ of strongly positively-rejected solutes and over-estimates the CP for the negatively-rejected ones. This observation is especially important for the interpretation of ion rejection from multi-ion solutions in nanofiltration where strong positive and pronounced negative rejections can occur simultaneously for solutes of different charges.

We conclude that for reliable interpretation of pressure-driven membrane measurements it is desirable to reduce the inhomogeneity of $\mathrm{CP}$ distribution to a minimum in membrane-testing devices.

Keywords: concentration polarization, inhomogeneous distribution, unstirred-layer thickness, pressure-driven, spacer-filled feed channel. 


\section{Introduction}

Correct description of concentration polarization (CP) is important for the interpretation of experimental data with the purpose of obtaining accurate information on the membrane properties and predicting properly the performance of pressure-driven processes. The classical approach to quantify CP is based on the socalled Nernst model [1-5], which postulates the existence of a diffusion boundary layer sharply separated from a perfectly-stirred core-flow zone. Correlations deduced from heat and mass transfer (typically kinetics of dissolution) experiments are frequently used to estimate the thickness of boundary layer. The experimental output of such studies is the dependences of dissolution rate on the cross-flow velocity, diffusion coefficient of the solute (or thermal conductivity in the heat-transfer studies) as well as on the flow-channel geometry. Evidently, this can provide information only on massor heat-transfer characteristics averaged along the channel.

Due to the complexity of the phenomena, computational fluid dynamics (CFD) has recently become a technique of choice for the calculation of flow and concentration fields inside feed membrane channels. Thus for instance some studies simulated the flow and CP in narrow rectangular channels with spacers [6-11]. Their results show inhomogeneous $\mathrm{CP}$ distribution strongly dependent on the spacer configuration and reveal up to an order of magnitude variation in the local mass-transfer coefficient depending on the position along the membrane surface $[10,12-14]$.

Despite this pronounced inhomogeneity, the results of CFD simulations are often reported as Sherwood number (or mass-transfer coefficient) averaged over the membrane surface. Sherwood number is defined as a ratio of a characteristic length (for example, channel height) and the unstirred-layer thickness. Some authors made reference to the error associated with an inaccurate description in the CP boundary layer in determining the intrinsic rejection and the membrane transport parameters [15]. However, these estimates related only to the specific experimental conditions of those studies. More systematically, [16] simulated numerically the distribution of solute concentration at the membrane surface in infinitely-broad slit-like channels with developed laminar flow and the membrane exhibiting constant rejection (independent of either solute concentration or trans-membrane volume flow). The results were interpreted in terms of dependence of average extent of CP on the trans-membrane mass-transfer rate. Mostly, empirical correlations were considered but some comparison with the film (Nernst) model was also performed. It was found that at weak CPs the film model reproduced the results of numerical simulations quite well but at moderate to strong CPs there were considerable deviations. The authors ascribed them to the effect of "suction" due to the non-zero trans-membrane volume flow, which is often disregarded. However, we believe that actually a major part of those deviations occurred due to the inhomogeneity of distribution of $\mathrm{CP}$ along the 
channel. Unfortunately, only the case of $100 \%$ solute rejection was considered in the context of comparison with the film model.

In a number of cases instead of the rejecting-membrane boundary condition, the authors used the impermeable dissolving wall condition, which sets a constant solute concentration and zero normal fluid velocity at the wall $[12,17,18]$. In this case, the contribution of more stagnant zones to the average mass-transfer (dissolution) rate is under-proportional to their surface fraction since the local Sherwood number is smaller in those regions. In the more realistic case of membrane boundary condition, this contribution is over-proportional at positive rejections due to the stronger solute accumulation. In any case, the use of dissolving-wall boundary condition does not allow for an analysis of dependence of averaged mass transfer on the solute rejection. This dependence can be essential as we will see below. In addition, even in the studies where the membrane boundary condition was used [7-10,13,14,19,20], reporting the results in terms of averaged Sherwood number (instead of reciprocal one) makes difficult drawing conclusions on the correlation between the averaged mass transfer and solute diffusion coefficient (especially when the CP is not weak). In turn, a previous study characterized shear stresses and mass transfer coefficients at the membrane surface of a high pressure stirred filtration cell at different conditions [21]. The authors observed a considerable variation of local values of shear stress at relatively low rotation speeds, with its averaged standard deviation decreasing with increasing Reynolds number. As for the mass-transfer coefficient, the radial distribution is qualitatively similar but more uniform than shear stress, although there is still a variation around $50 \%$ in the time averaged Sherwood number.

Fluid-dynamics simulations are quite complex mathematically and can be performed only numerically. In order to obtain transparent analytical results, in this study we adopt an alternative approach: we postulate a distribution of unstirred-layer thickness over the membrane surface and use a simple locally-1D description of CP. Its simplicity enables us to use the membrane boundary condition with various intrinsic solute rejections (including negative ones). In this way, we obtain local permeate concentration as a function of trans-membrane volume flux and solute diffusion coefficient. Then, the observable rejection is calculated via averaging this concentration over the membrane surface by using the postulated probability-density function for the distribution of unstirred-layer thickness.

Of course, this approach is approximate primarily because it disregards lateral solute flows (both convective and diffusive). However, if the unstirred-layer thickness is much smaller than the characteristic scale of inhomogeneity of its distribution along the membrane surface the local 1D approach can be sufficiently accurate. This condition can be met for typical feed spacers with the characteristic mesh sizes of a couple of 
millimeters, while the typical unstirred-layer thickness in laminar flows is around 100 $\mu \mathrm{m}$. Some of the results obtained below do not depend on the exact form of distribution function. For more quantitative illustrations in the first instance, we will use a simple log normal distribution. We will also test our approach in the case of a distribution function obtained from CFD simulations [18].

\section{Theory}

First, we define the classical convection-diffusion equation in the unstirred layer for the $1 \mathrm{D}$ description of local CP:

$I_{v}=-D \frac{d c}{d x}+J_{v} c$

where $I_{s}$ is the trans-membrane solute flux, $D$ is the solute diffusion coefficient, $c$ is the solute concentration, $x$ is the coordinate across the unstirred layer, $I v$ is the transmembrane volume flux. The boundary condition is a given solute concentration, $c_{f}$, at the external boundary of unstirred layer (local Nernst model).

Further, it is assumed that there is a distribution of thickness of unstirred layer over the membrane surface. Taking into account that there is a most probable value of this thickness, $\delta$, and defining the ratio of actual local unstirred-layer thickness to the most probable one as the parameter $\alpha$, Eq. (1) can be rewritten in this way:

$\alpha \cdot f_{s}=-\frac{d c}{d \xi}+\alpha \cdot P_{e} \cdot c$

where

$f_{s} \equiv \frac{I_{s} 0}{D}$

$P_{e} \equiv \frac{D_{v} \delta}{D}$

$\xi \equiv{ }^{x} / 0$

By assuming that the solute rejection is a constant independent of solute concentration and trans-membrane volume flow, Eq. (2) can be easily integrated to yield the solute concentration at the membrane surface, $c_{m}$ :

$c_{m}=\frac{c_{f}}{1-R+R \cdot \exp \left(-\alpha \cdot P^{\theta}\right)}$

(6) 
where $c_{f}$ is the feed concentration and $R$ is the solute rejection defined as $\frac{\left(C_{m}-C_{p}\right)}{C_{m}}$. Therefore for the local permeate concentration, $c_{p}$, we obtain

$$
c_{p}=(1-R) \cdot c_{m} \equiv \frac{(1-R) \cdot c_{f}}{1-R+R \cdot \exp (-\alpha \cdot P Q)}
$$

(7)

The averaged permeate concentration is:

$$
\bar{c}_{p}-(1-R) \cdot c_{f} \cdot \int_{0}^{\infty} \frac{f(\alpha) d \alpha}{1-R+R \cdot \exp \left(-\alpha \cdot P_{e}\right)}
$$

where $f(c)$ is the probability-density function for the distribution of relative unstirredlayer thickness (scaled on its most probable value, $\delta$ ) over the membrane surface. By definition,

\section{$\int_{0}^{\infty} f(\alpha) d \alpha \equiv \mathbf{1}$}

One can also use Eq. (7) to define an effective value of parameter $\alpha$ that would correspond to the average permeate concentration if the inhomogeneous distribution of unstirred-layer thickness over the membrane surface were disregarded:

$$
c_{p}=\frac{(1-R) \cdot c_{f}}{1-R+R \cdot \exp \left(-\alpha_{\theta f f} \cdot P_{Q}\right)}
$$

Such procedure corresponds to neglecting the distribution of extent of $\mathrm{CP}$ over the membrane surface and describing $\mathrm{CP}$ by using a single value of an effective unstirredlayer thickness (or mass-transfer coefficient).

Finally, by using Eq. (8), for $\alpha_{\text {fff }}$ we obtain

$$
\alpha_{\theta f f}=-\frac{1}{P_{\theta}} \cdot \ln \left[1+\frac{1}{R} \cdot\left(\frac{1}{\rho_{\theta}^{\infty} \frac{f(\alpha) d \sigma}{1-R+R+e x p\left(-\alpha \cdot P_{\theta}\right)}}-1\right)\right]
$$

Expectedly, such effective value turns out a function of solute rejection, Péclet number $(P e)$, and distribution function. Below, we will investigate these dependences for a model probability-distribution function and demonstrate that neglecting the distribution of $\mathrm{CP}$ extent over the membrane surface can lead to considerable errors in the interpretation of experimental data, especially in the case of mixed solutions containing components with essentially different rejections (e.g., ions of different charge magnitude in nanofiltration). 


\section{Results and Discussion}

First of all, let us consider the limiting case of weak CP. By using Eq. (11) one can show that at $\mathrm{Pe} \rightarrow 0$ (weak CP)

$x_{\theta f f} \underset{P_{\theta \rightarrow 0}}{\longrightarrow} \alpha_{m} \equiv \int_{0}^{\infty} a \cdot f f a r d a$

which is just the definition of average value of parameter $\alpha$. This is expectable because the weak $\mathrm{CP}$ is linear in the Péclet number so the surface concentration is controlled just by the average unstirred-layer thickness over the membrane surface.

Notably, in the academic literature it is customary to report the results of CFD simulations of mass transfer (e.g., in spacer-filled channels) in terms of average Sherwood number $[17,18,22]$. The latter is inversely proportional to the unstirred-layer thickness. In our terms, this would correspond to the averaging of reciprocal parameter $\alpha$ that is to the calculation of its harmonic average according to:

$\alpha_{h \omega} \equiv \frac{1}{\int_{0}^{\infty} \frac{f(\alpha)}{\alpha} d \alpha}$

According to Eq. (12), the correct asymptotics at weak CP corresponds to the arithmetic average of relative unstirred-layer thickness. Fig. 1 shows the ratio of harmonic average to the arithmetic average as a function of the width parameter of the log-normal distribution used below for the sample calculations. 


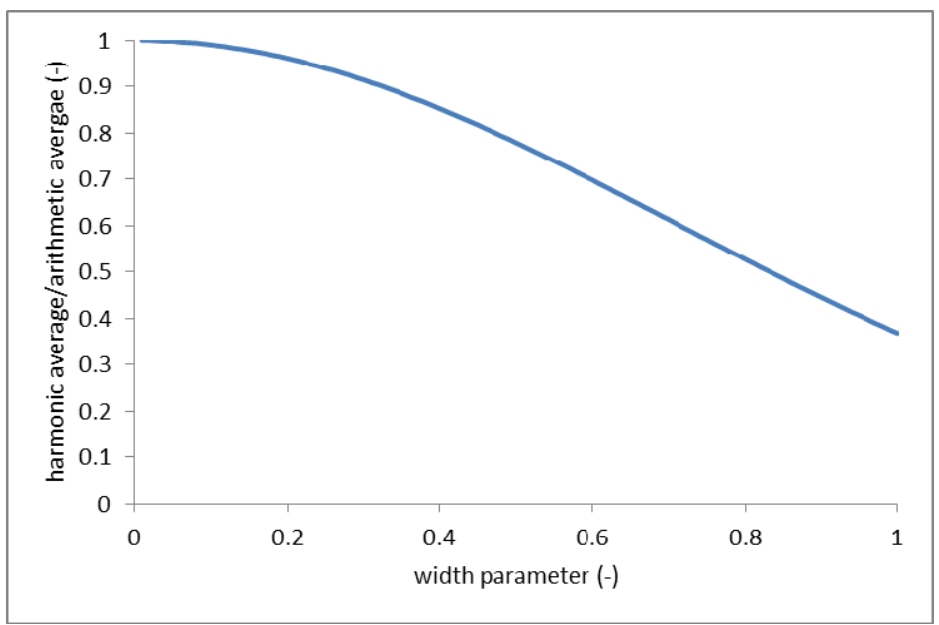

Fig. 1. Ratio of harmonic average to the arithmetic average as a function of the width parameter of the log-normal distribution

Expectedly, this ratio tends to unity when the width parameter goes to zero. However, at any finite width the harmonic average is smaller than the arithmetic one. This means that the averaging of Sherwood number underestimates the average impact of even weak inhomogeneous CP. The harmonic average unduly amplifies the contribution of zones with the weakest CP. One can see that at the width parameter equal to one (relatively broad but still rather compact distribution, see Fig. 2), the averaging of Sherwood number underestimates the extent of weak CP by a factor of almost 3.

The ratio plotted in Fig. 1 was obtained by using a specific probability-density function. By assuming a sufficiently narrow distribution we will show that this ratio is always smaller than one even for an arbitrary distribution function. Indeed, one can always represent parameter $\alpha$ as the sum of its arithmetic average and deviation from it

$\alpha \equiv \alpha_{m}+\delta \alpha$

By substituting Eq. (14) into the definition of harmonic average of Eq. (13), taking into account that $\int_{0}^{\infty} \delta \alpha \cdot f(\alpha) d \alpha=0$ (by definition) and $\delta \alpha_{\alpha} / \alpha_{m} \ll 1$ (narrow distribution), one can show that

$\alpha_{h \sigma} \approx \alpha_{m}-\frac{1}{\alpha_{m}} \cdot \int_{0}^{\infty}(\delta \alpha)^{2} \cdot f(\alpha) d \alpha$ 
The integral in the right-hand side of Eq. (15) is always positive so $\alpha_{m a} \leqslant \alpha_{m}$.

At finite Péclet numbers even the arithmetic averaging is incorrect. We will illustrate this for a model distribution of unstirred-layer thickness. Let us use a particular case of log-normal distribution given by this equation

$$
f(\omega)=\frac{1}{\alpha=\sigma \sqrt{2 \pi}}+\operatorname{sxp}\left[-\frac{\left(\ln u(x)-\nu^{2}\right)^{2}}{2 \sigma^{2}}\right]
$$

where $\sigma$ is the width parameter. With this version of log-normal distribution the maximum probability density always occurs at $\alpha=\mathbf{1}$. This is illustrated by Fig. 2, which also shows that the dependence of function shape on the width parameter is quite strong. Thus for instance, at $\sigma=\mathbf{1}$ the distribution is much broader than at $\tau=0.5$. Nonetheless, even in the former case the fraction of zones where the unstirred-layer thickness is ca.10 times larger than the most probable value is about $7 \%$. For $\sigma=0.75$ this fraction is below $1 \%$, which seems to correspond (by the order of magnitude) to the color maps published in [23]. Interestingly, the function of Eq. (16) generates exactly the same distribution of the reciprocal parameter $\alpha$ (proportional to the Sherwood number, see above). Therefore, the graphs from Fig. 2 can be directly compared with the CFD-generated probability-density distributions of local Sherwood number shown in Fig. 9 of [18] which look qualitatively similar (especially for the larger Schmidt numbers).

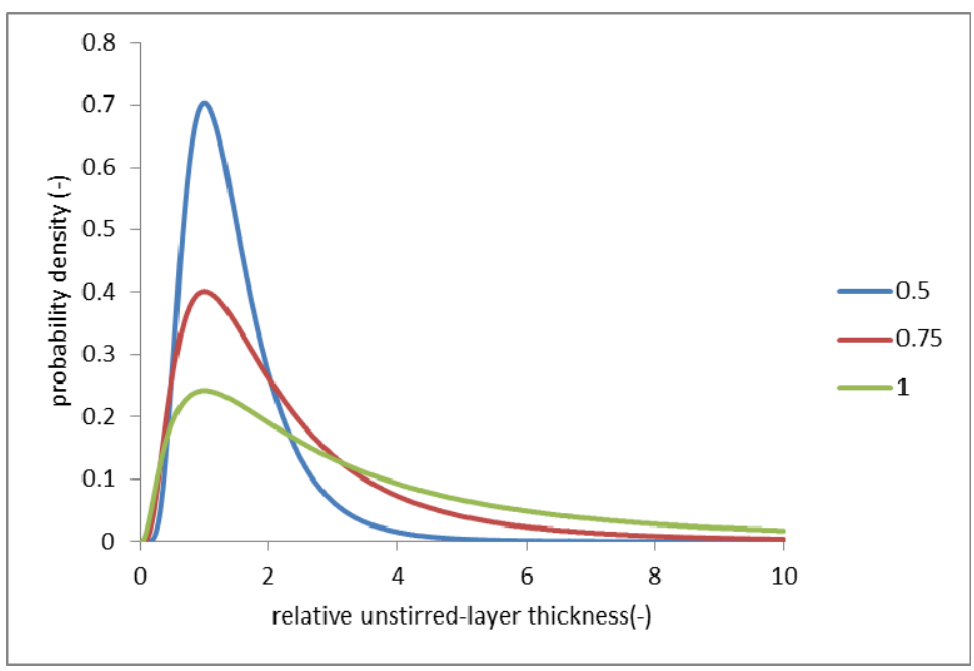

Fig. 2. The probability-density distribution functions used for the sample calculations below; the legend gives the values of width parameter.

Fig. 3 shows the dependences of the ratio of effective relative unstirred-layer thickness defined by Eq.(11) and the average one defined by Eq.(12) on the Péclet number (extent of $\mathrm{CP}$ ). The deviation of this ratio from unity is a measure of error introduced due to neglecting the $\mathrm{CP}$ distribution over the membrane surface. As discussed above, 
all the curves converge to one at $P \theta \rightarrow 0$ (weak $\mathrm{CP}$ ), which means that the procedure of averaging the unstirred-layer thickness (but not of Sherwood number) is correct in this limiting case. At finite Péclet numbers, there are considerable deviations from unity. Remarkably, they strongly depend on the solute rejection and the Péclet number (and through the latter on the solute diffusivity). Most interestingly, the ratio is larger than one for high positive rejections whereas it is smaller than one for the negative rejections. This can be explained by the fact that (due to the exponential nature of $\mathrm{CP}$ ) the strongly-rejected solutes over-proportionally accumulate within poorly-stirred zones (larger unstirred-layer thicknesses) so their average concentration at the membrane surface turns out larger than predicted with the average unstirred-layer thickness. At the same time, the concentration of negatively-rejected solutes at the membrane surface is reduced and tends to zero at very strong CP. Therefore, poorlystirred zones make under-proportional contribution to the trans-membrane flux of such solutes. To sum up, our analysis shows that disregarding CP distribution over the membrane surface under-estimates the $\mathrm{CP}$ of strongly positively-rejected solutes and over-estimates the $\mathrm{CP}$ for the negatively-rejected ones. Fig. 3 shows that the difference in the effective thicknesses of unstirred layer between the highest positive and the negative rejections can be as large as four to six times for the broader distributions. It is also important to note the considerable dependence on the Péclet number, which depends on the trans-membrane volume flow and the solute diffusion coefficient.
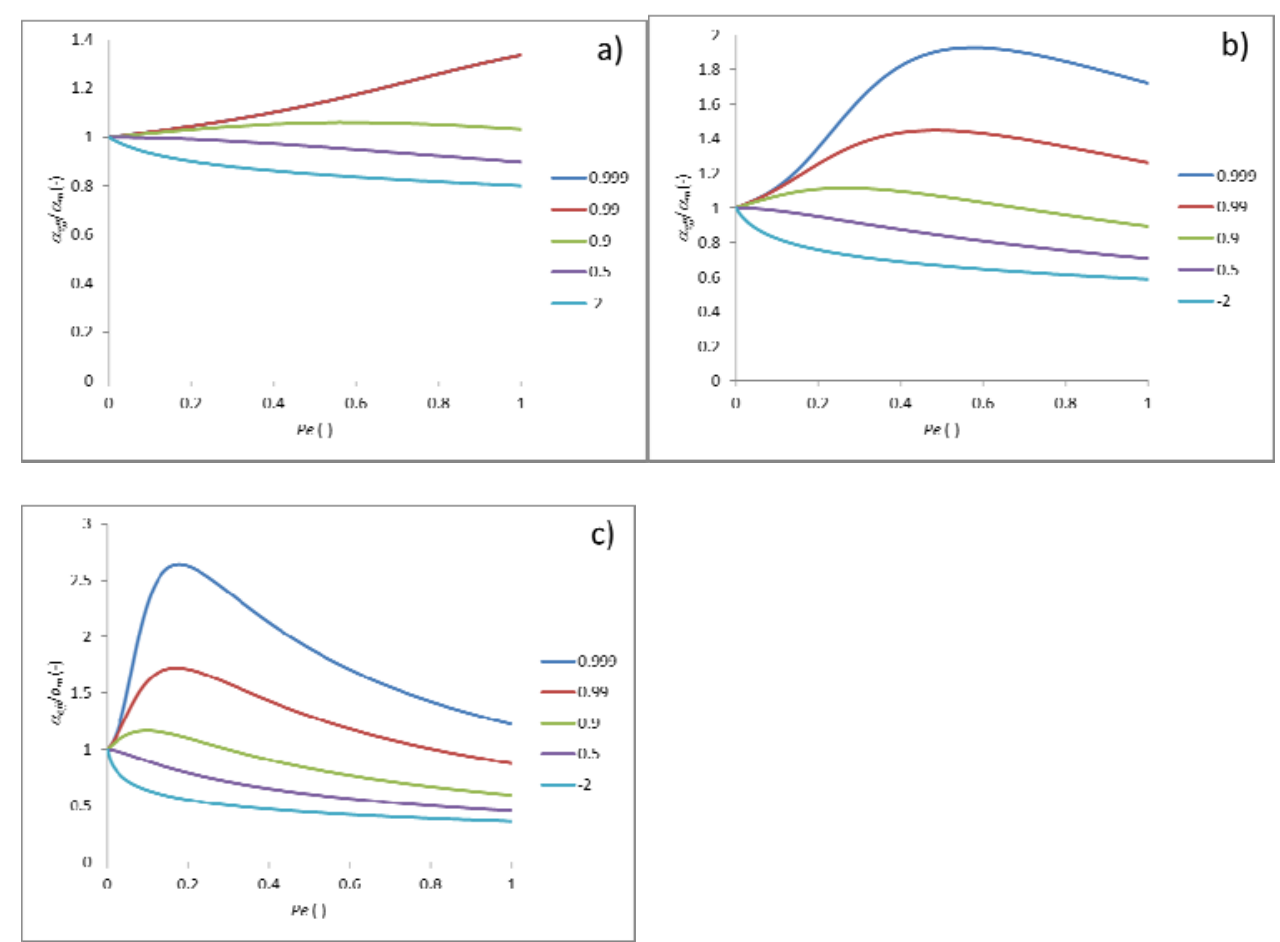

Fig. 3. Ratio of effective to the average unstirred-layer thickness vs. Péclet number for various intrinsic rejections and width parameters: (a) $\sigma=0.5$; (b) $\sigma=0.75$ and (c) $\sigma=1$ 
Even with a single solute this is a problem because one cannot quantitatively fit the dependence of rejection on the trans-membrane volume flow by using a single value of unstirred-layer thickness (or mass-transfer coefficient). However, the situation gets even more problematic with several solutes experiencing very different intrinsic rejections. A relevant example is the nanofiltration of multi-ionic solutions containing, for example, sulfates and chlorides. The intrinsic rejection of sulfates in NF is typically above $99 \%$ whereas chloride ions in the presence of sulfates often experience pronounced negative rejections. According to our analysis, with inhomogeneous CP, one cannot use the same effective thickness of unstirred layer for these two solutes even approximately. In [24] a procedure was suggested of fitting the unstirred-layer thickness to the dependence of rejection of dominant salt on the trans-membrane volume flow and using this thickness to make the $\mathrm{CP}$ corrections for the trace ions. The present analysis reveals that this procedure may well be incorrect in the case of strongly-rejected dominant salts and negatively-rejected trace ions especially if the measurements are done in test cells with spacer-filled feed channels as it was the case in [24]. Besides, our results probably can explain the dependence of estimated thickness of unstirred layer on the type of dominant salt observed in [24]. The fitted thickness was larger for salts with lower diffusion coefficients, which would correspond to the dependence of Péclet number on the solute diffusion coefficient to the left from the maxima seen in Fig.3. Additionally, the dependence of effective unstirred-layer thickness on the intrinsic solute rejection could also contribute because salts with lower diffusion coefficients had higher intrinsic rejections.

Now we will use one of the CFD-generated probability-density distributions of local Sherwood number shown in Fig. 16 of $[18,25]$. Notably, the largest Schmidt number in these simulations was 100, which is not representative of typical solutes in aqueous solutions (this low value of Schmidt number was probably used because of computational reasons; the realistic value is around 1000). Moreover, in this distribution there is a non-zero probability of Sherwood number is equal exactly to zero. Within the scope of our locally 1D model this rise to infinitely strong concentration polarization. Therefore, this point was discarded and the rest of distribution was rescaled to have all the probabilities to add up to $100 \%$.

Fig. 4 shows the effective unstirred-layer thickness (defined by Eq(11) scaled on the average one (defined by $\mathrm{Eq}(12)$ as a function of Péclet number defined with the average thickness. Qualitatively the results are similar to those obtained for the broader log-normal distribution (Fig.3c). However, the deviations from unity are even more pronounced in this case. The reason is that the CFD-generated distribution is still broader primarily due to the existence of a range of quite small Sherwood numbers occurring with roughly equal probability density of around $0.5 \%$. 
This shows that irrespective of the specific form of probability distribution function (if sufficiently broad) the concept of average unstirred-layer thickness becomes rather useless because it does not reflect the considerable dependence of overall concentration polarization on the intrinsic solute rejection, diffusion coefficient and trans-membrane volume flow.

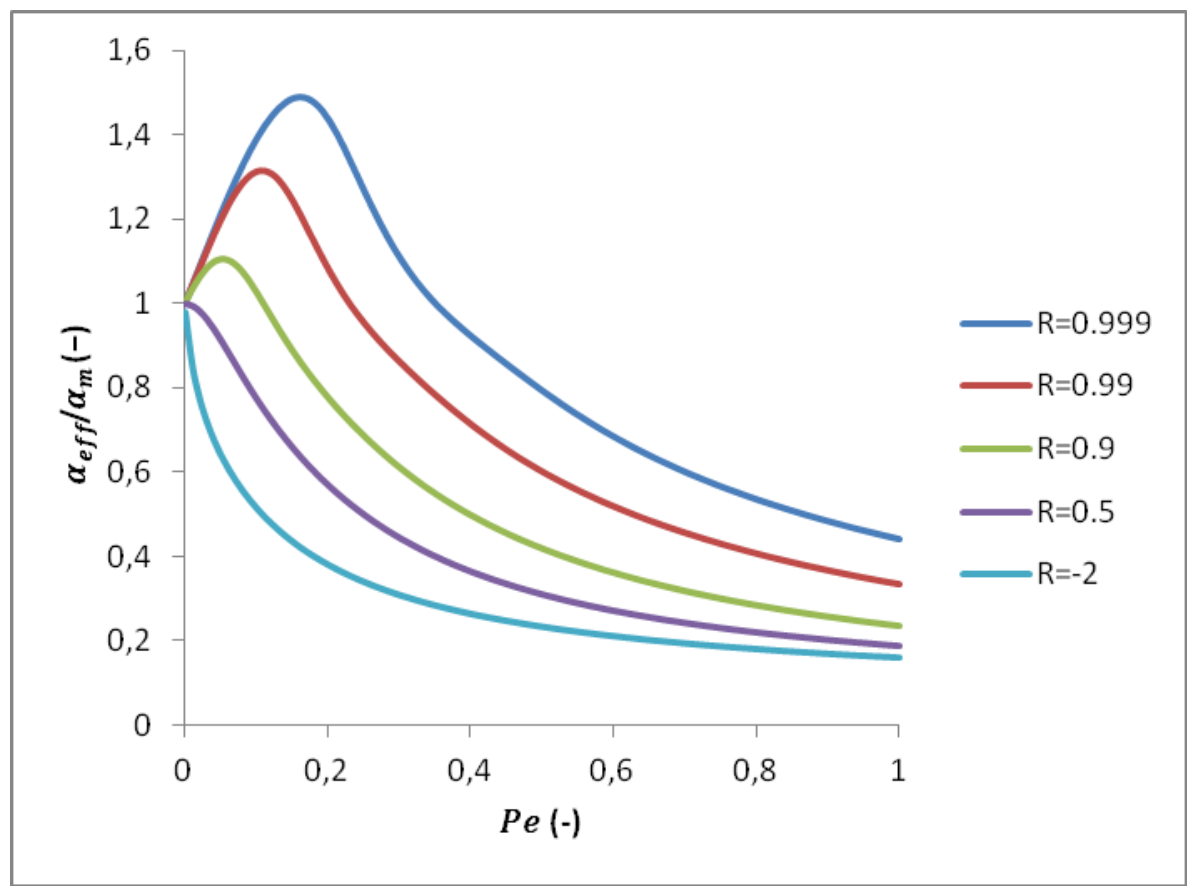

Fig. 4. Ratio of effective to the average unstirred-layer thickness vs. Péclet number for various intrinsic rejections for the case of CFD-generated distribution function

\section{Conclusions}

A number of CFD studies have demonstrated that there is a considerable distribution of extent of CP over the membrane surface especially in spacer-filled feed channels. Despite the up to an order of magnitude variability in the simulated local Sherwood number, the impact of this distribution on the observable rejections received little attention in the previous studies.

This study elucidates qualitative consequences of inhomogeneous CP by using a locally 1D description of $\mathrm{CP}$ and postulated probability distributions of unstirred-layer thickness over the membrane surface. The principal conclusions of our analysis are:

1. In the limiting case of weak CP averaging local unstirred-layer thickness produces correct results. On the contrary, averaging local Sherwood number (inversely proportional to the unstirred-layer thickness) is incorrect even in this limiting case.

2. At moderate to strong $\mathrm{CP}$, the whole description in terms of a single value of Nernst- layer thickness fails because the effective unstirred-layer thickness turns 
out a noticeable function of intrinsic rejection, trans-membrane volume flow and solute diffusion coefficient.

3. Disregarding the $\mathrm{CP}$ distribution under-estimates the $\mathrm{CP}$ of strongly positivelyrejected solutes and over-estimates the $\mathrm{CP}$ for the negatively-rejected ones; the difference in the effective unstirred-layer thicknesses calculated for such solutes can be several times.

4. Inhomogeneous CP complicates considerably the task of obtaining reliable information on the membrane transport properties from experimental data. Therefore, in membrane-testing devices it is desirable to reduce this inhomogeneity to a minimum.

\section{Acknowledgement}

The authors gratefully appreciate the numerical data provided by A.J. Karabelas and acknowledge support from the project "Valorization of by-product from industrial effluents: integration of membrane technologies in the separation, concentration and purification steps" (Waste2Product, CTM2014-57302-R) funded by the Ministry of Science and Innovation (MINECO, Spain) and the Catalan government (project ref.2014SGR050).

\section{List of symbols}

c: solute concentration

$c_{f}:$ feed solute concentration (external boundary of unstirred layer)

$c_{m}$ : solute concentration at the membrane surface

$c_{p}$ : local permeate concentration

$\bar{c}_{p}$ : average permeate concentration

$D$ : solute diffusion coefficient

$f(\alpha)$ : probability-density function of the distribution of relative unstirred-layer thickness (scaled on its most probable value, $\delta$ )

$I_{s}:$ trans-membrane solute flow

$I_{v}$ : trans-membrane volume flux 
Pe: Péclet number

$R$ : solute intrinsic rejection

$x$ : coordinate across the unstirred layer

Greek letters

$\alpha$ : ratio of actual local unstirred-layer thickness to the most probable one

$\alpha_{e f f}$ : effective value of parameter $\alpha$

$\alpha_{h a}:$ harmonic average of parameter $\alpha$

$\alpha_{m}:$ arithmetic average of parameter $\alpha$

$\delta:$ most probable thickness of unstirred layer

$\delta \alpha$ : deviation of parameter $\alpha$

$\xi$ : coordinate normalized by the local unstirred layer thickness

$\tau$ : width parameter

\section{References}

[1] S. Bhattacharjee, J.C. Chen, M. Elimelech, Coupled model of concentration polarization and pore transport in crossflow nanofiltration, AIChE J. 47 (2001) 2733-2745. doi:10.1002/aic.690471213.

[2] S. Bouranene, P. Fievet, A. Szymczyk, M. El-Hadi Samar, A. Vidonne, Influence of operating conditions on the rejection of cobalt and lead ions in aqueous solutions by a nanofiltration polyamide membrane, J. Memb. Sci. 325 (2008) 150-157. doi:10.1016/j.memsci.2008.07.018.

[3] W.R. Bowen, B. Cassey, P. Jones, D.L. Oatley, Modelling the performance of membrane nanofiltration - Application to an industrially relevant separation, J. Memb. Sci. 242 (2004) 211-220. doi:10.1016/j.memsci.2004.04.028.

[4] V. Geraldes, M.D. Afonso, Prediction of the concentration polarization in the nanofiltration/reverse osmosis of dilute multi-ionic solutions, J. Memb. Sci. 300 (2007) 20-27. doi:10.1016/j.memsci.2007.04.025.

[5] T.Y. Qiu, P.A. Davies, Concentration polarization model of spiral-wound membrane modules with application to batch-mode RO desalination of brackish water, Desalination. 368 (2015) 36-47. doi:10.1016/j.desal.2014.12.048. 
[6] V. Geraldes, V. Semião, M.N. de Pinho, Flow management in nanofiltration spiral wound modules with ladder-type spacers, J. Memb. Sci. 203 (2002) 87-102. doi:10.1016/S0376-7388(01)00753-0.

[7] V. Geraldes, V. Semião, M.N. de Pinho, The effect of the ladder-type spacers configuration in NF spiral-wound modules on the concentration boundary layers disruption, Desalination. 146 (2002) 187-194. doi:10.1016/S00119164(02)00467-8.

[8] V. Geraldes, V. Semiao, M. Norberta de Pinho, Concentration polarisation and flow structure within nanofiltration spiral-wound modules with ladder-type spacers, Comput. Struct. 82 (2004) 1561-1568. doi:10.1016/j.compstruc.2004.03.052.

[9] L. Song, S. Ma, Numerical studies of the impact of spacer geometry on concentration polarization in spiral wound membrane modules, Ind. Eng. Chem. Res. 44 (2005) 7638-7645. doi:10.1021/ie048795w.

[10] M. Amokrane, D. Sadaoui, M. Dudeck, C.P. Koutsou, New spacer designs for the performance improvement of the zigzag spacer configuration in spiral-wound membrane modules, Desalin. Water Treat. 3994 (2015) 1-9.

doi:10.1080/19443994.2015.1022003.

[11] M. Paipuri, S.H. Kim, O. Hassan, N. Hilal, K. Morgan, Numerical modelling of concentration polarisation and cake formation in membrane filtration processes, Desalination. 365 (2015) 151-159. doi:10.1016/j.desal.2015.02.022.

[12] J. Schwinge, D.E. Wiley, D.F. Fletcher, Simulation of the flow around spacer filaments between channel walls. 2. Mass-transfer enhancement, Ind. Eng. Chem. Res. 41 (2002) 4879-4888. http://www.scopus.com/inward/record.url?eid=2-s2.00037130790\&partnerID=tZOtx3y1.

[13] T. Ishigami, H. Matsuyama, Numerical Modeling of Concentration Polarization in Spacer-filled Channel with Permeation across Reverse Osmosis Membrane, Ind. Eng. Chem. Res. 54 (2015) 1665-1674. doi:10.1021/ie5039665.

[14] M. Amokrane, D. Sadaoui, C.P. Koutsou, A.J. Karabelas, M. Dudeck, A study of flow field and concentration polarization evolution in membrane channels with two-dimensional spacers during water desalination, J. Memb. Sci. 477 (2015) 139-150. doi:10.1016/j.memsci.2014.11.029.

[15] S. Bason, V. Freger, Phenomenological analysis of transport of mono- and divalent ions in nanofiltration, J. Memb. Sci. 360 (2010) 389-396. doi:10.1016/j.memsci.2010.05.037.

[16] V. Geraldes, M.D. Afonso, Generalized mass-transfer correction factor for nanofiltration and reverse osmosis, AIChE J. 52 (2006) 3353-3362. 
doi:10.1002/aic.10968.

[17] J.L.C. Santos, V. Geraldes, S. Velizarov, J.G. Crespo, Investigation of flow patterns and mass transfer in membrane module channels filled with flow-aligned spacers using computational fluid dynamics (CFD), J. Memb. Sci. 305 (2007) 103117. doi:10.1016/j.memsci.2007.07.036.

[18] C.P. Koutsou, S.G. Yiantsios, a. J. Karabelas, A numerical and experimental study of mass transfer in spacer-filled channels: Effects of spacer geometrical characteristics and Schmidt number, J. Memb. Sci. 326 (2009) 234-251. doi:10.1016/j.memsci.2008.10.007.

[19] J.M. Miranda, J.B.L.M. Campos, Mass transfer in the vicinity of a separation membrane - The applicability of the stagnant film theory, J. Memb. Sci. 202 (2002) 137-150. doi:10.1016/S0376-7388(01)00747-5.

[20] V. Geraldes, V. Semião, M. Norberta Pinho, Hydrodynamics and concentration polarization in NF/RO spiral-wound modules with ladder-type spacers, Desalination. 157 (2003) 395-402. doi:10.1016/S0011-9164(03)00422-3.

[21] C.P. Koutsou, A.J. Karabelas, Shear stresses and mass transfer at the base of a stirred filtration cell and corresponding conditions in narrow channels with spacers, J. Memb. Sci. 399-400 (2012) 60-72. doi:10.1016/j.memsci.2012.01.029.

[22] E. Lyster, Y. Cohen, Numerical study of concentration polarization in a rectangular reverse osmosis membrane channel: Permeate flux variation and hydrodynamic end effects, J. Memb. Sci. 303 (2007) 140-153. doi:10.1016/j.memsci.2007.07.003.

[23] J.L.C. Santos, J.G. Crespo, V. Geraldes, OpenFOAM simulation of mass transfer in spacer-filled channels, in: Eur. Conf. Comput. Fluid Dyn., 2010: pp. 14-17. http://web.univ-ubs.fr/limatb/EG2M/Disc_Seminaire/ECCOMASCFD2010/papers/01454.pdf.

[24] A. Yaroshchuk, X. Martínez-Lladó, L. Llenas, M. Rovira, J. de Pablo, Solutiondiffusion-film model for the description of pressure-driven trans-membrane transfer of electrolyte mixtures: One dominant salt and trace ions, J. Memb. Sci. 368 (2011) 192-201. doi:10.1016/j.memsci.2010.11.037.

[25] A. J. Karabelas, 2015 (personal communication) 